\title{
Ion Acceleration by Flux Transfer Events in the Terrestrial Magnetosheath
}

\author{
Järvinen, $\mathrm{R}$.
}

2018-02-28

Järvinen , R, Vainio , R, Palmroth , M , Juusola , L, Hoilijoki , S , Pfau-Kempf , Y , Ganse , $U$, Turc , L \& von Alfthan , S 2018 , ' Ion Acceleration by Flux Transfer Events in the Terrestrial Magnetosheath ' Geophysical Research Letters, vol. 45 , no. 4 , pp. 1723-1731. DOI: 10.1002/2017GL076192

http://hdl.handle.net/10138/234758

https://doi.org/10.1002/2017GL076192

Downloaded from Helda, University of Helsinki institutional repository.

This is an electronic reprint of the original article.

This reprint may differ from the original in pagination and typographic detail.

Please cite the original version. 


\section{Geophysical Research Letters}

\section{RESEARCH LETTER \\ 10.1002/2017GL076192 \\ Ion Acceleration by Flux Transfer Events in the Terrestrial Magnetosheath}

Key Points:

- Global hybrid-Vlasov model predicts ion acceleration in the terrestrial magnetosheath

- The acceleration is associated with bow waves driven by propagating flux transfer events

- Velocity distributions of energetic ions are similar to observed magnetosheath ion injections

Supporting Information:

- Supporting Information S1

- Movie S1

- Movie S2

Correspondence to:

R. Jarvinen,

riku.jarvinen@fmi.fi

\section{Citation:}

Jarvinen, R., Vainio, R., Palmroth, M., Juusola, L., Hoilijoki, S., Pfau-Kempf, Y., ... von Alfthan, S. (2018). Ion acceleration by flux transfer events in the terrestrial magnetosheath. Geophysical Research Letters, 45, 1723-1731. https://doi.org/10.1002/2017GL076192

Received 27 OCT 2017 Accepted 29 JAN 2018 Accepted article online 6 FEB 2018 Published online 20 FEB 2018
@2018. American Geophysical Union. All Rights Reserved.

\author{
R. Jarvinen' ${ }^{1}$, R. Vainio ${ }^{2}$, M. Palmroth ${ }^{3}$, L. Juusola1 ${ }^{1}$, S. Hoilijoki ${ }^{3,4}$ (D) Y. Pfau-Kempf ${ }^{3}$, \\ U. Ganse ${ }^{3}$ iD , L. Turc ${ }^{3}$, and S. von Alfthan ${ }^{5}$
}

${ }^{1}$ Finnish Meteorological Institute, Helsinki, Finland, ${ }^{2}$ Department of Physics and Astronomy, University of Turku, Turku, Finland, ${ }^{3}$ Department of Physics, University of Helsinki, Helsinki, Finland, ${ }^{4}$ Now at Laboratory for Atmospheric and Space Physics, University of Colorado Boulder, Boulder, CO, USA, ${ }^{5}$ CSC, IT Center for Science, Espoo, Finland

\section{Introduction}

Energetic ions are common in Earth's near space including the region controlled by the terrestrial magnetic field, the magnetosphere, and the magnetosheath, which is the domain of shocked solar wind surrounding the magnetosphere (Lucek et al., 2005). The magnetosheath forms when the supersonic, magnetized solar wind plasma flow encounters the geomagnetic field resulting in the creation of the bow shock, slowing down and heating of the flow, compressing the terrestrial magnetic field earthward on the dayside and stretching of the magnetosphere into a magnetotail at the nightside. The bow shock is the outer boundary of the magnetosheath, and the magnetopause separates the magnetosheath from the magnetosphere. Most energetic charged particles accelerated around Earth are associated with acceleration at the magnetopause or in the magnetosphere (Marcucci et al., 2004; Paschmann et al., 1979; Sibeck et al., 1987) or near the bow shock (Armstrong et al., 1985; Chang et al., 2000; Louarn et al., 2003). All of these populations are found in the magnetosheath via diffusion or reconnection through the magnetopause or transport from the bow shock.

Observations of accelerated magnetosheath particles include time-energy dispersive energetic ion injections termed Magnetosheath Dispersed Signatures (MDS) measured by the Cluster spacecraft (Louarn et al., 2003). In an injection event higher-energy particles appear before lower-energy particles reach the instrument. During a period of 2 months more than $20 \mathrm{MDS}$ events were identified, where the ions at the energy of $30 \mathrm{keV}$ typically arrived about $100 \mathrm{~s}$ before the ions at the energy of $5 \mathrm{keV}$. Velocity distributions of energetic ions in the MDS events are ring like. The origin of the events is unclear. Some were related to discontinuities in the upstream solar wind and were interpreted in terms of the time of flight effects and the motion of the acceleration source region at the bow shock. In other cases upstream transients were less clearly coincident with the injections, suggesting that the origin of the MDS events could be some magnetospheric process.

Flux transfer events (FTEs) are associated with time-dependent magnetic reconnection between the interplanetary magnetic field (IMF) embedded in the turbulent magnetosheath plasma and the geomagnetic field at the dayside magnetopause (Dungey, 1961; Russell \& Elphic, 1979a; Southwood et al., 1988). A characteristic signature of an FTE is a bipolar magnetic field structure and a cross-sectional radius from half to few Earth radii in the direction normal to the magnetopause (Raeder, 2006). In global simulations of Earth's solar wind interaction FTEs originate as magnetic flux ropes or magnetic islands propagating from the dayside to the nightside near the magnetopause (Hoilijoki et al., 2017; Omidi \& Sibeck, 2007; Raeder, 2006). 
Passages of the FTEs in Earth's polar region are associated with energetic ion injections occurring in the magnetospheric cusps, where the geomagnetic field lines are open to the magnetosheath (Lockwood \& Smith, 1989; Omidi \& Sibeck, 2007; Sibeck \& Omidi, 2012).

In this work we analyze ion acceleration driven by the FTEs as a possible source of the magnetosheath ion injections using a two-dimensional polar plane simulation run made with the global hybrid-Vlasov model called Vlasiator (von Alfthan et al., 2014). In the model FTEs drive fast-mode magnetosheath bow waves resulting in populations of energetic ions (Pfau-Kempf et al., 2016).

The study is organized as follows. In the next section we briefly describe the simulation model and the analyzed run. Then we present the modeling results and discuss and compare them to in situ spacecraft observations. At the end we summarize our findings.

\section{Model}

Vlasiator is a global hybrid-Vlasov simulation of Earth's solar wind interaction (von Alfthan et al., 2014). The simulation models ions directly by representing the time evolution of a velocity distribution by the Vlasov equation, while electrons are a charge-neutralizing fluid. The magnetic field is propagated by Faraday's law, and the electron momentum equation (Ohm's law) defines the electric field. The analyzed run is 2D3Vdimensional covering the two-dimensional spatial polar plane and three-dimensional ion and electron velocities and electromagnetic field quantities. In the simulation a planet-centered coordinate system similar to the Geocentric Solar Ecliptic (GSE) coordinate system is used. The $x$ axis is toward the incident solar wind flow injected from the front wall, the $z$ axis is along the geomagnetic field at the $x$ axis, and the periodic $y$-direction completes the right-handed coordinate system.

The simulation run analyzed in this work is a steady-driven fast solar wind and southward IMF case. The incident upstream solar wind ion density, velocity, temperature, and IMF are $n_{\mathrm{sW}}=1 \mathrm{~cm}^{-3}, \vec{V}_{\mathrm{sW}}=(-750,0,0) \mathrm{km} / \mathrm{s}$, $T_{\text {sw }}=5 \times 10^{5} \mathrm{~K}$, and $\vec{B}_{\mathrm{sw}}=(0,0,-5) \mathrm{nT}$, respectively. The solar wind ions are protons. Earth's magnetic field is represented by a two-dimensional line dipole (Daldorff et al., 2014). The run has $3,000 \times 2,400\left(n_{x} \times n_{z}\right)$ spatial Cartesian cubic grid cells with a grid cell side length of $300 \mathrm{~km}$. The simulation domain extends from $x=-94 R_{E}$ to $48 R_{E}$ and $z=-56 R_{E}$ to $56 R_{E}$, where the radius of Earth $R_{E}=6,371 \mathrm{~km}$. The inner boundary is at an altitude of $4.7 R_{E}$. The velocity grid in each spatial cell has $268^{3}$ cells for phase space from $-4,020 \mathrm{~km} / \mathrm{s}$ to $4,020 \mathrm{~km} / \mathrm{s}$ in all three dimensions. A side length of a velocity grid cell is $30 \mathrm{~km} / \mathrm{s}$. Velocity cells with lowest phase space density are neglected according to a sparsity condition. See von Alfthan et al. (2014) for details of the Vlasiator model and Hoilijoki et al. (2017) for more details of the analyzed run.

Figure 1 displays different regions of Earth's solar wind interaction and FTEs formed at the dayside magnetopause in the analyzed simulation run. The tail of the magnetosphere is not plotted as we focus on magnetosheath processes in this work. See Palmroth et al. (2017) for analysis of the tail processes.

\section{Results}

Figure 2 and an animation in the supporting information (Movie S1 in the supporting information) show an FTE (ellipse-shaped high ion parallel temperature region) created at the subsolar magnetopause due to reconnection at multiple X lines (Raeder, 2006; Omidi \& Sibeck, 2007) modulated by the magnetosheath fluctuations (Hoilijoki et al., 2017). A fast-mode bow wave is initiated when the speed of the FTE reaches about $250 \mathrm{~km} / \mathrm{s}$ (Figure 2a and animation at $t \sim 1,835 \mathrm{~s}$ ) (Pfau-Kempf et al., 2016). The bow wave soon extends from the earthside of the FTE to the dayside magnetosheath (Figure 2c). Upon becoming detached from the dayside magnetopause and entering the cusp region, the FTE reaches its maximum velocity of $450 \mathrm{~km} / \mathrm{s}$. In the cusp region the FTE undergoes secondary reconnection with the open magnetic field lines and decelerates (Sibeck \& Omidi, 2012) from $t=1,870 \mathrm{~s}$ to 1,920 s. The FTE leaves the cusp region after $t \sim 1,900 \mathrm{~s}$ to higher latitudes propagating again near antiparallel to the magnetic field at the nightside magnetopause with velocity $\sim 340 \mathrm{~km} / \mathrm{s}$ (Figure $2 \mathrm{~d}$ ). As the propagation proceeds the bow wave extends far into the magnetosheath (Figure 2e). The FTE grows weaker in the nightside magnetosheath due to reconnection against the magnetopause but survives to tens of Earth radii downstream (Eastwood et al., 2012).

Elevated ion parallel temperatures in Figures $2 c-2 e$ near the magnetic island and the bow wave indicate ion acceleration associated with the propagation of the FTE. The acceleration starts when the magnetic island reaches the cusp region. Reconnection jets originate from the magnetospheric side of the FTE and orient 


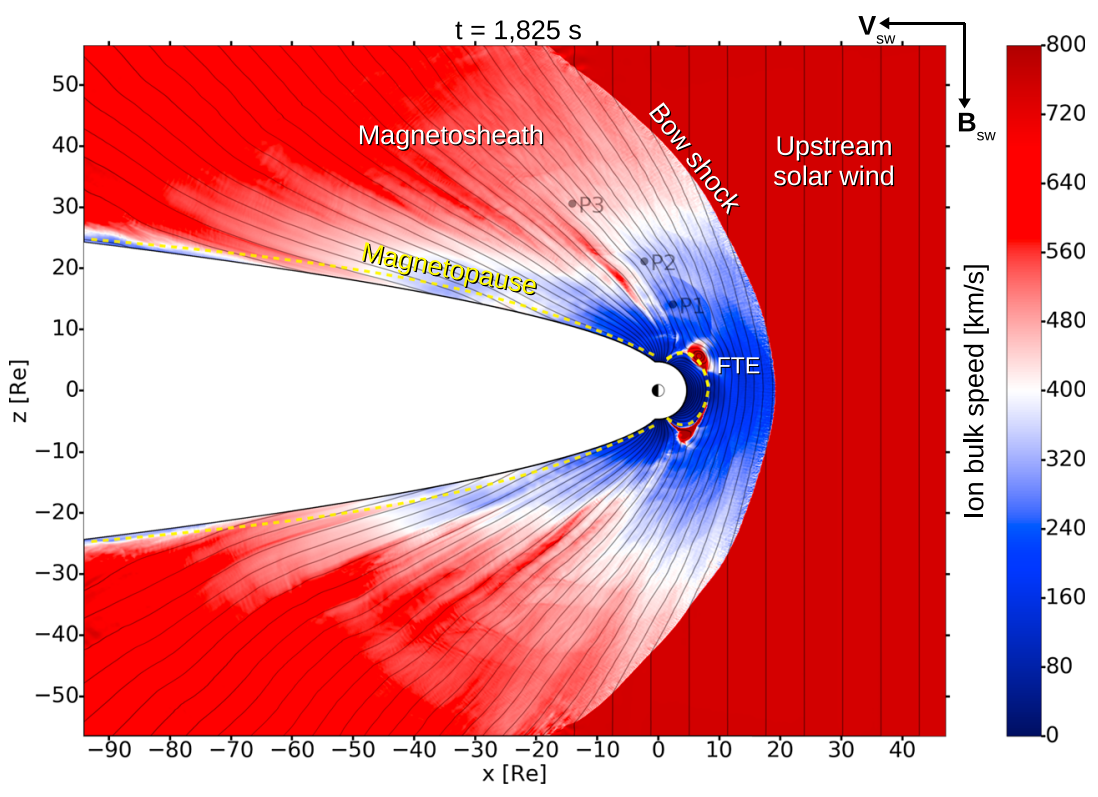

Figure 1. A snapshot of the magnitude of the ion bulk velocity and magnetic field lines in the global hybrid-Vlasov simulation run. Denoted are the upstream solar wind, the bow shock, and the magnetosheath. Yellow dashed curves indicate the approximate magnetopause location. The $\vec{V}_{s w}$ and $\vec{B}_{s w}$ arrows give the orientation of the upstream solar wind velocity and interplanetary magnetic field, respectively. The circle in the origin has the radius of Earth with white and black halves indicating the dayside and nightside hemispheres. Points P1-P3 are used to extract ion velocity distributions and time series of ion energy spectra in Figures 4 and 5.

nearly ahead and behind of the magnetic island along its motion at the magnetopause. In the cusp region the jets get intensified and are directed more perpendicular than parallel to the FTE motion due to the reconnection with the open vertical magnetic field. Reconnection accelerates ions at the magnetic field lines passing in the vicinity of the magnetic island in the magnetospheric side, which is seen as elevated ion parallel temperatures ahead of the FTE in the Front \#1 region of Figure 2e (red, magenta, and white colors within the dashed curve).

In addition to the Front \#1 reconnection region, ion acceleration occurs at the magnetic field lines not connected near the magnetic island but passing through the bow wave. This region where elevated ion parallel temperature occurs ahead of the bow wave is marked as Front \#2 in Figure 2e. The morphology of the bow wave with its precursor front resembles the curved bow shock and the foreshock. In the middle magnetosheath the magnetic field is nearly aligned with the bow wave normal (Field line \#2 in Figure 2e) resulting in a quasi-parallel configuration favorable to ion acceleration and upstream reflection. Closer to the bow shock, the magnetosheath bow wave turns toward a quasi-perpendicular configuration, which is less efficient for ion acceleration.

Elevated ion parallel temperatures occur independently at the bow wave and the reconnection region since the two regions are magnetically mostly unconnected from each other. However, interactions between ions accelerated in the reconnection and at the bow wave are possible at the interface of the two regions. The Alfvén velocity is of the order of few hundred kilometer per second in the cusp region (Figure S4 in the supporting information), and thus, ions with the energies of $10-30 \mathrm{keV}$ cannot be produced by the reconnection alone. Some of the ions in the reconnection jet from the cusp region are clearly injected for further acceleration at the bow wave near the interface between the two acceleration regions in Figure $2 \mathrm{c}$ at $x=3 R_{E}$ and $z=9 R_{E}$. Moreover, an accelerated ion population arising from another FTE (in the cusp region at $t \sim 1,930 \mathrm{~s}$ ) is injected and accelerated in the bow wave of the preceding FTE displaying an interaction between ion acceleration by multiple FTEs (Movie S1 in the supporting information at $t \sim 2,010 \mathrm{~s}, x=-5 R_{E}$, and $z=23 R_{E}$ ).

Note that, in addition to the analyzed FTE, also minor FTEs initiate bow waves in the simulation (Figure 2 and Movie S1). The minor FTEs decay in the cusp region due to the secondary reconnection (Sibeck \& Omidi, 2012), 

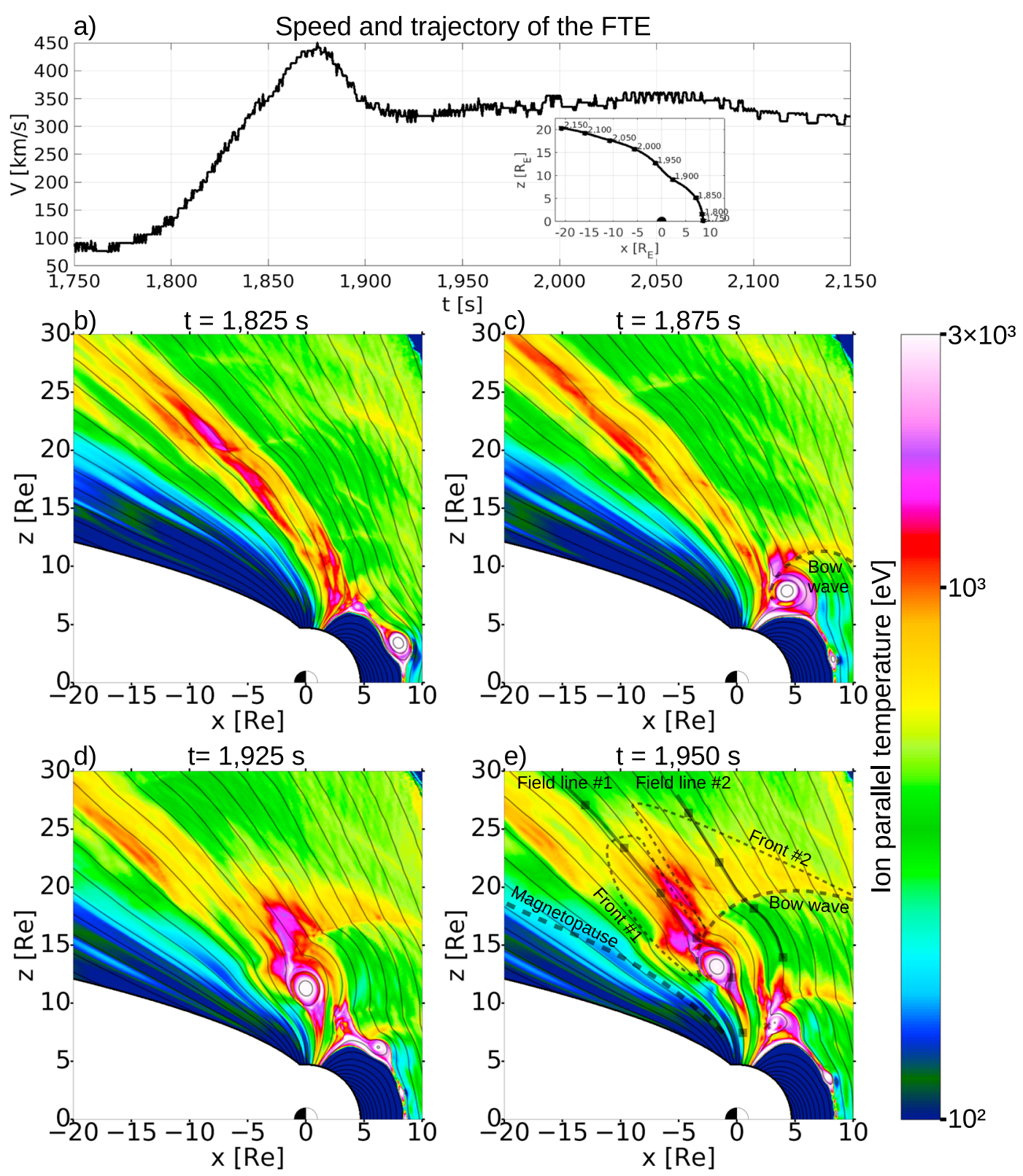

Figure 2. Motion of the flux transfer event (FTE) and time evolution of the ion parallel temperature and magnetic field lines in the northern magnetosheath. (a) The velocity and trajectory of the FTE as a function of time, which is seen in the (b) -(e) panels as a white circular region moving from the dayside to the nightside. The velocity is a $10 \mathrm{~s}$ running average. The trajectory of the FTE is shown in the small plot with simulation times $50 \mathrm{~s}$ apart marked as spheres along the path. (b-e) Snapshots of the ion parallel temperature in the northern magnetosheath at four different simulation times. Elevated values in the plot are associated with ion acceleration along the magnetic field. The circle in the origin has the radius of Earth with white and black halves indicating the dayside and nightside hemispheres. Approximate locations of the bow wave, the magnetopause, and the accelerated ion populations are marked with dashed lines and curves. Panel (e) also shows two magnetic field lines in bold with squares $5 R_{E}$ apart along the field lines, which are used to extract plasma temperatures in Figure 3. Average locations of the magnetic reconnection points associated with two FTEs are denoted by $X$ in the last panel. See an animation of the ion parallel temperatures and magnetic field lines (Movie S1) and a version of the figure without overlaid markings in the supporting information (Figure S3). 

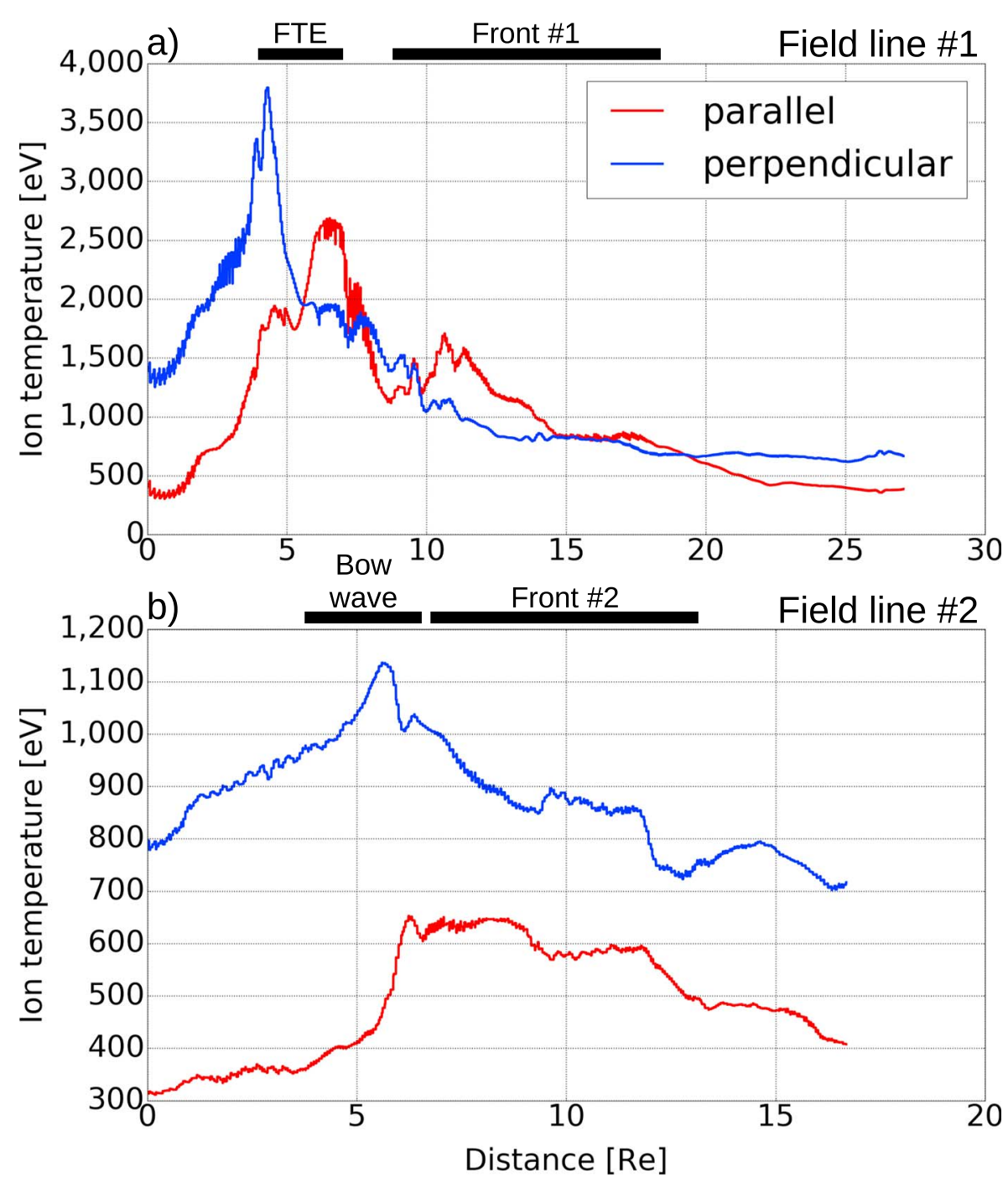

Figure 3. Traces of the ion parallel and perpendicular temperatures along two magnetic fields lines crossing near (a) the flux transfer event (FTE) and (b) the bow wave at simulation time $t=1,950 \mathrm{~s}$. The field lines are shown in Figure 2e. Field line \#1 passes near the magnetic island, while Field line \#2 passes the bow wave away from the magnetic island. Bars above the plots denote the regions of accelerated ions ahead of the magnetic island (Front \#1) and the bow wave (Front \#2) as well as the FTE and the bow wave themselves as marked in Figure 2e. See a version of the figure including additional bulk parameters in the supporting information (Figure S5).

but their bow waves propagate in the magnetosheath. Further, the minor FTEs result in enhanced ion parallel temperatures in the high-latitude magnetosheath near the magnetopause without signatures of magnetic islands (e.g., Figure 2b).

Figure 3 shows ion temperatures at magnetic field lines at the bow wave and the reconnection (magnetic island) regions. Both ion parallel $\left(T_{\text {par }}\right)$ and perpendicular $\left(T_{\text {perp }}\right)$ temperatures show the strongest increase along the field line near the magnetic island (Field line \#1 in Figure 2e), with $T_{\text {perp }}$ steeply increasing up to $\sim 3,800 \mathrm{eV}$ and $T_{\text {par }}$ up to $\sim 2,600 \mathrm{eV}$, compared to the background values of $T_{\text {perp }} \sim 700 \mathrm{eV}$ and $T_{\text {par }} \sim 400 \mathrm{eV}$ (Figure 3a). $T_{\text {par }}$ is elevated to around $800-1,600 \mathrm{eV}$ ahead of the reconnecting FTE near the nightside magnetopause. In the middle magnetosheath (Field line \#2 in Figure 2e) the temperatures sharply increase at the bow wave from their background values of $T_{\text {perp }} \sim 800 \mathrm{eV}$ and $T_{\text {par }} \sim 350 \mathrm{eV}$ up to $T_{\text {perp }} \sim 1,100 \mathrm{eV}$ and $T_{\text {par }} \sim 650 \mathrm{eV}$, respectively (Figure 3b). The population ahead of the bow wave (Front \#2 in Figure 2e) has $T_{\text {par }} \sim 600 \mathrm{eV}$.

Acceleration processes are analyzed in more detail in Figure 4, where ion velocity distributions in the simulation are shown. Cuts of the distributions are plotted in directions parallel $\left(V_{\text {par }}\right)$ and perpendicular $\left(V_{\text {perp1,2 }}\right)$ 


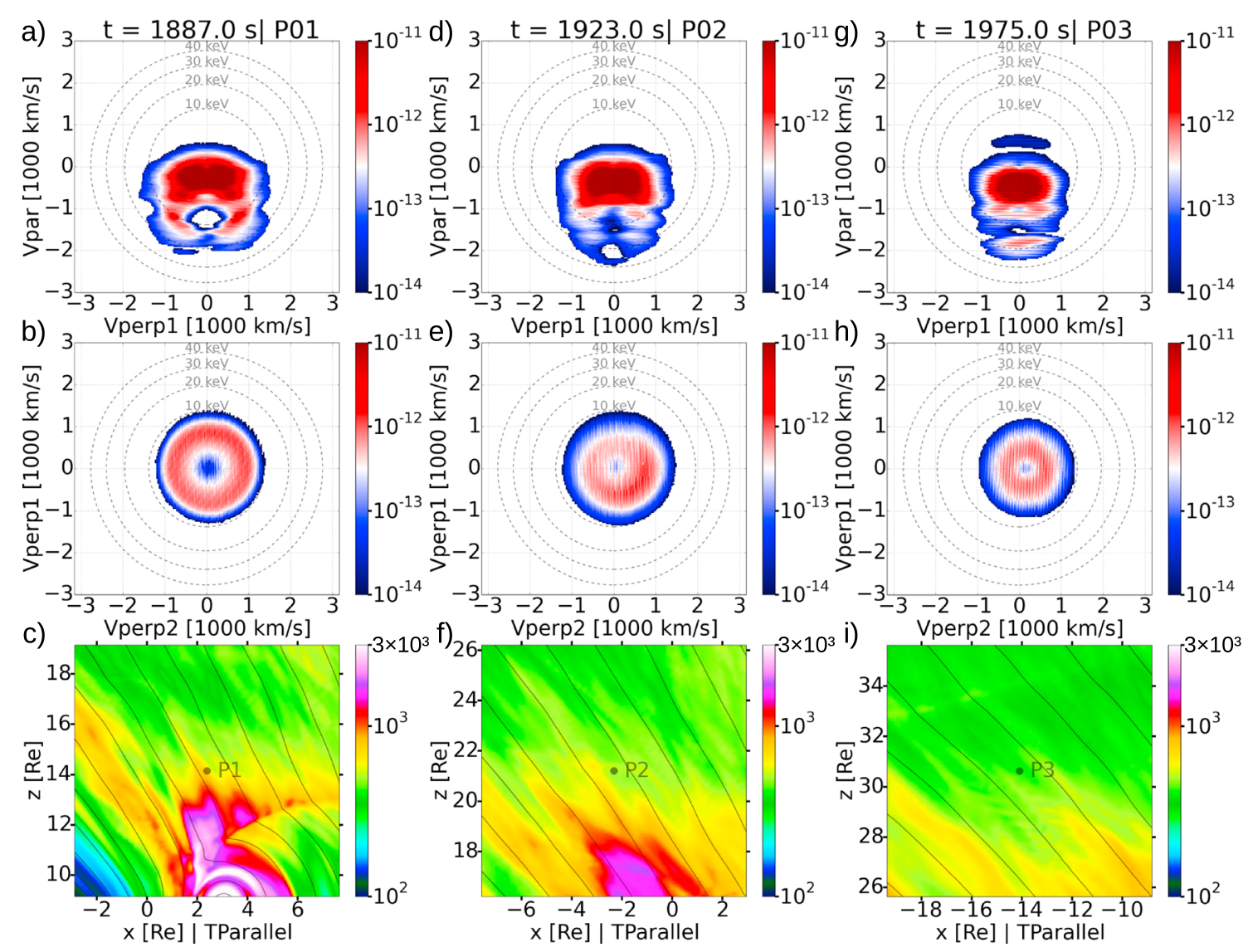

Figure 4. Snapshots of the ion $(a, b, d, e, g, h)$ velocity distributions and $(c, f, i)$ parallel temperatures at three points in the northern magnetosheath. The points are as follows: $\mathrm{P} 1=\left(2.4 R_{E}, 14.2 R_{E}\right), \mathrm{P} 2=\left(-2.3 R_{E}, 21.2 R_{E}\right)$, and $\mathrm{P} 3=\left(-14.1 R_{E}, 30.6 R_{E}\right)$ as shown in Figure 1 . The simulation times of the velocity distributions are $t=1,887 \mathrm{~s}(\mathrm{P} 1), t=1,923 \mathrm{~s}(\mathrm{P} 2)$, and $t=1,975 \mathrm{~s}(\mathrm{P} 3)$, which were chosen to coincide when the ion population accelerated by the flux transfer event reaches each point. The velocities are plotted in the coordinate system defined by the magnetic field, with $V_{\text {par }}$ along the magnetic field and the $V_{\text {perp } 1}$ and $V_{\text {perp2 }}$ perpendicular to the magnetic field. The velocity plots are two-dimensional cuts of the three-dimensional distribution. Top row $\left(V_{\text {perp } 1}, V_{\text {par }}\right)$ is centered around zero $\left(V_{\text {perp2 }}=-100 \ldots 100 \mathrm{~km} / \mathrm{s}\right)$, and the middle row is centered at $V_{\text {par }}=-1,000 \mathrm{~km} / \mathrm{s}(-1,100 \ldots-900 \mathrm{~km} / \mathrm{s})$ to make them comparable to observations (Louarn et al., 2003). Coloring of the top and middle rows gives the phase space density in each $(30 \mathrm{~km} / \mathrm{s})^{3} \mathrm{bin}$. See an animated version of the figure in the supporting information (Movie S2).

to the magnetic field in similar format as the observations presented by Louarn et al. (2003). Figure 4 shows three points P1-P3 in the northern magnetosheath at the arrival of the FTE bow wave precursor (Front \#2 in Figure 2e). The bulk population of the shocked solar wind is visible as the strong flux maximum at velocities below $1,000 \mathrm{~km} / \mathrm{s}$ flowing in the negative $V_{\text {par }}$ direction at all three points. Accelerated ions at energies exceeding $5 \mathrm{keV}$ are seen as local flux maxima on both sides of the negative $V_{\text {par }}$ direction. As there are only few particles at pitch angles aligned with the magnetic field, the accelerated population forms a ring perpendicular to the magnetic field or a hollow cone in three dimensions as discussed by Louarn et al. (2003).

The accelerated population shows clear signatures of a shock drift-like acceleration process (in addition to Figure 4 see also Movie S2 in the supporting information) (Armstrong et al., 1985; Louarn et al., 2003). The ring or hollow cone distribution corresponds to a reflected ion beam in the bow wave precursor front (Figures 4a, $4 \mathrm{~d}, 4 \mathrm{~g}$ ), which is responsible for the increased parallel temperature in the corresponding moment description (Figure $3 b$ ). When the precursor front crosses the point, the beam disappears and the distribution shows signatures of perpendicular acceleration, consistent with the increase of the perpendicular temperature in the compressed magnetic field of the wave (Figures S5 and S7-S9 in the supporting information). The shock drift-like accelerated population forms a hollow cone in the first place before pitch angle scattering moves ions from the reflected beam also to pitch angles aligned with the magnetic field. Ions distributed on the hollow cone in the velocity space around the magnetic field at velocities well above the bulk solar wind population resemble the observed magnetosheath ion injections (Louarn et al., 2003). 


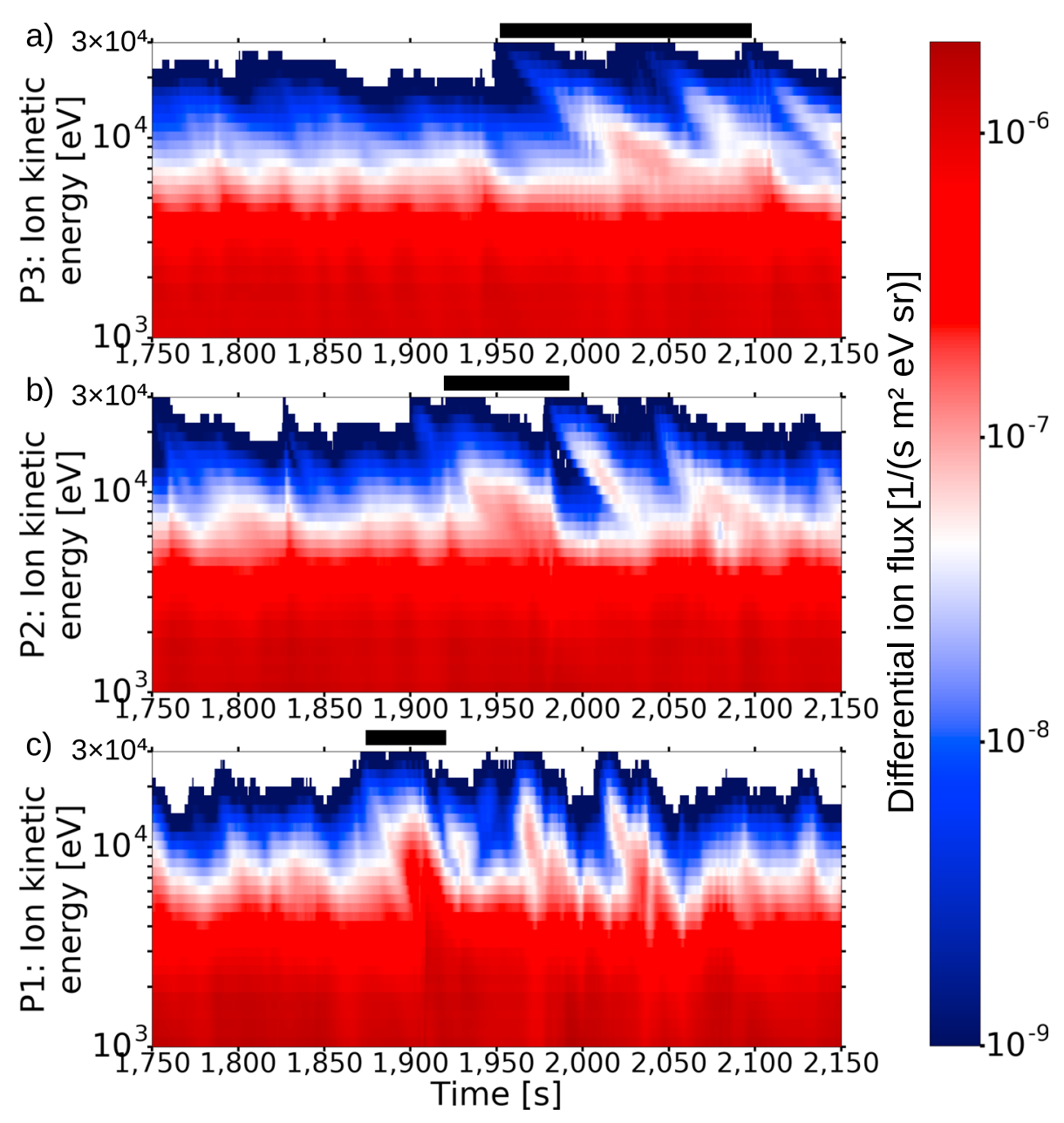

Figure 5. $(\mathrm{a}-\mathrm{c})$ lon time-energy spectrograms in three points in the northern magnetosheath. The points $\mathrm{P} 1-\mathrm{P} 3$ are the same as in Figure 4. Coloring is the omnidirectional differential ion flux determined as $\sum_{i}\left(f_{i} v_{i}\right) /(4 \pi \Delta E)$, where the summation is over all velocity cells in the energy range $[E, E+\Delta E]$ within the spatial cell where the point is located and $f_{i}$ and $v_{i}$ are the ion phase space density and speed in a velocity cell $i$, respectively. Bars above the plots denote the energy-time dispersed energetic ion injection caused by the ion acceleration associated with the flux transfer event and the bow wave.

Note that multiple reflected ion beams are visible as multiple local maxima on both sides of the negative $V_{\text {par }}$ for example, in Figure $4 \mathrm{~d}$. They can be related to the magnetosheath fluctuations, which modulate the structure of the bow waves, or be associated with multiple bow waves propagating in the magnetosheath. Further, accelerated ions up to $\sim 20 \mathrm{keV}$ with a flux maximum centered at pitch angles aligned with the magnetic field are also evident in the simulation (see Figure S6 and Movie S2 in the supporting information). These events occur when an ion beam from the secondary reconnection of a minor FTE in the cusp region is injected from the downstream to the bow wave. The difference to the shock drift-like acceleration described in the previous paragraph is that in this case there occurs no reflected ion beam at the flux maximum at high energies. When the downstream beam crosses the bow wave, the beam may undergo acceleration by electric field associated with the bow wave (see, e.g., Dimmock et al., 2011; Kan et al., 1991, and references therein).

Velocity distributions analyzed by Louarn et al. (2003) are from the dusk hemisphere in the dayside northern magnetosheath $\left(x_{\mathrm{GSE}} \sim 5 R_{E}, y_{\mathrm{GSE}} \sim 7 R_{E}\right.$, and $z_{\mathrm{GSE}} \sim 9 R_{E}$ ). For comparison, out of our analyzed points, P1 is in the dayside hemisphere (solar-zenith angle $<90^{\circ}$ ), while P2 and P3 are in the nightside hemisphere (solar-zenith angle $>90^{\circ}$ ). The IMF in Louarn et al. (2003) case is mostly along the $y$ axis, which results in the accelerated ion population centered in the direction approximately parallel to the magnetic field $\left(V_{\text {par }}>0\right)$. In our simulation case the IMF is southward resulting in the shock drift-like accelerated ion population 
centered around the direction antiparallel to the magnetic field $\left(V_{\mathrm{par}}<0\right)$. The simulation covers the polar plane meaning that possible effects related to the duskward location of the observations from the $y_{\mathrm{GSE}}=0$ plane, like the dawn-dusk component of the FTE motion, cannot be resolved.

Note that a gyroradius of a proton moving at the bulk speed in the magnetosheath in this simulation case is of the order of $100 \mathrm{~km}$, which is smaller than the grid cell side length. However, ion gyromotion is fully resolved in the velocity space in the model. Resolving spatial ion gyroscales is not essential here since we are not considering, for instance, ion-cyclotron resonant wave-particle interactions. Further, protons at energies of $10 \mathrm{keV}$ and above have gyroradii several times larger than the grid cell side length in the magnetosheath.

Figure 5 shows simulated virtual spacecraft ion time-energy spectra in the points P1-P3 during the FTE bow wave precursor front crossings. Ion injections are evident as higher-energy particles arrive before lower energies. The slope of the injection is steeper in the points close to the cusp region, where protons start to get accelerated, compared to the points away from the cusp region. Farther away in the magnetosheath, the higher-energy ions have traveled farther ahead of the FTE and the bow wave resulting in a decreasing slope of the injection. Further, the interactions between energetic ion populations from different FTEs and their bow waves are visible in P3 as multiple injections occurring in the same time at $t=1,950 \ldots 2,150 \mathrm{~s}$. Ion fluxes of the injection events decrease farther away as the energetic populations have expanded over a larger region. The duration of the energetic proton injection associated with the analyzed FTE from $30 \mathrm{keV}$ to $5 \mathrm{keV}$ ions lasts about 1-2 min, which is very similar to the observed value during the MDS events (Louarn et al., 2003).

\section{Conclusions}

We have reported shock drift-like ion acceleration at the bow waves propagating ahead of the FTEs in the terrestrial magnetosheath in a global two-dimensional hybrid-Vlasov polar plane simulation of Earth's solar wind interaction. In the analysis interactions between reconnection and bow wave accelerated ions were also seen. Energetic proton populations resulting from the acceleration in the magnetosheath reach the energy of $30 \mathrm{keV}$ in the simulation, and their velocity distributions resemble time-energy dispersive ion injections observed by the Cluster spacecraft in the magnetosheath. Thus, processes within the magnetosheath are a potential source of energetic ions near Earth in addition to acceleration in the magnetosphere and the solar wind transient interaction with the bow shock.

A conjunction of magnetospheric spacecraft like Cluster and Magnetospheric Multiscale (MMS) is called for to investigate this issue based on in situ observations. The searched signals should include energetic ion populations when the solar wind transient and magnetospheric origin of the acceleration can be ruled out as well as a direct detection of the bow waves associated with the FTEs in the magnetosheath. Further investigations should include detailed ion dynamics in the magnetosheath acceleration, the spatial structure and motion of the FTE bow waves in three dimensions, and statistics of the locations and velocity distributions of the observed MDS events with respect to the upstream IMF orientation.

Acknowledgments

The work of R. J., R. V., M. P., L. J., S. H., Y. P-K., and U. G. was supported by the Academy of Finland (Hybrid-Vlasov consortium, grants 267144 and 267186) and the ERC Consolidator grant (ERC-CoG-682068-PRESTISSIMO). The work of L. T. was supported by a Marie Sklodowska-Curie Individual Fellowship (704681). The run was carried out using HLRS/Stuttgart Hazel Hen through PRACE Tier-0 resource grant (2014112573) from the 12th call. The analyses were performed on the Voima supercomputer at the Finnish Meteorological Institute. Vlasiator is an open source simulation with its source code available at https://github.com/fmihpc/vlasiator. The simulation data that support the findings of this study are available from the corresponding author upon reasonable request.
Finally, magnetic flux ropes and sheath regions occur in space plasma environments beyond Earth (Brain et al., 2010; Cloutier et al., 1999; Jasinski et al., 2016; Russell \& Elphic, 1979b; Slavin et al., 2012; Wei et al., 2010). Their role in the acceleration of charged particles should be further studied.

\section{References}

Armstrong, T. P., Pesses, M. E., \& Decker, R. B. (1985). Shock drift acceleration, Geophysical Monograph Series (Vol. 35, pp. 271-285). Washington, DC: American Geophysical Union. https://doi.org/10.1029/GM035p0271

Brain, D. A., Baker, A. H., Briggs, J., Eastwood, J. P., Halekas, J. S., \& Phan, T.-D. (2010). Episodic detachment of Martian crustal magnetic fields leading to bulk atmospheric plasma escape. Geophysical Research Letters, 37, L14108. https://doi.org/10.1029/2010GL043916

Chang, S.-W., Scudder, J. D., Fennell, J. F., Friedl, R., Lepping, R. P., Russell, C. T., ... Spence, H. E. (2000). Energetic magnetosheath ions connected to the Earth's bow shock: Possible source of cusp energetic ions. Journal of Geophysical Research, 105, 5471-5488. https://doi.org/10.1029/1999JA900468

Cloutier, P. A., Law, C. C., Crider, D. H., Walker, P. W., Chen, Y., Acuña, M. H., ... Ness, N. F. (1999). Venus-like interaction of the solar wind with Mars. Geophysical Research Letters, 26, 2685-2688. https://doi.org/10.1029/1999GL900591

Daldorff, L. K. S., Tóth, G., Gombosi, T. I., Lapenta, G., Amaya, J., Markidis, S., \& Brackbill, J. U. (2014). Two-way coupling of a global Hall magnetohydrodynamics model with a local implicit particle-in-cell model. Journal of Computational Physics, 268, $236-254$. https://doi.org/10.1016/j.jcp.2014.03.009

Dimmock, A. P., Balikhin, M. A., \& Hobara, Y. (2011). Comparison of three methods for the estimation of cross-shock electric potential using Cluster data. Annales Geophysicae, 29, 815-822. https://doi.org/10.5194/angeo-29-815-2011

Dungey, J. W. (1961). Interplanetary magnetic field and the auroral zones. Physical Review Letters, 6, 47-48. https://doi.org/10.1103/PhysRevLett.6.47 
Eastwood, J. P., Phan, T. D., Fear, R. C., Sibeck, D. G., Angelopoulos, V., Øleroset, M., \& Shay, M. A. (2012). Survival of flux transfer event (FTE) flux ropes far along the tail magnetopause. Journal of Geophysical Research, 117, A08222. https://doi.org/10.1029/2012JA017722

Hoilijoki, S., Ganse, U., Pfau-Kempf, Y., Cassak, P. A., Walsh, B. M., Hietala, H., ... Palmroth, M. (2017). Reconnection rates and X line motion at the magnetopause: Global 2D-3V hybrid-Vlasov simulation results. Journal of Geophysical Research: Space Physics, 122, $2877-2888$. https://doi.org/10.1002/2016JA023709

Jasinski, J. M., Slavin, J. A., Arridge, C. S., Poh, G., Jia, X., Sergis, N., ... Waite, J. H. (2016). Flux transfer event observation at Saturn's dayside magnetopause by the Cassini spacecraft. Geophysical Research Letters, 43, 6713-6723. https://doi.org/10.1002/2016GL069260

Kan, J. R., Lyu, L. H., \& Mandt, M. E. (1991). Quasi-parallel collisionless shocks. Space Science Reviews, 57, $201-236$. https://doi.org/10.1007/BF00216045

Lockwood, M., \& Smith, M. F. (1989). Low-altitude signatures of the cusp and flux transfer events. Geophysical Research Letters, 16, 879-882. https://doi.org/10.1029/GL016i008p00879

Louarn, P., Budnik, E., Sauvaud, J. A., Parks, G., Meziane, K., Bosqued, J. M., ... Amata, E. (2003). Observation of energy-time dispersed ion structures in the magnetosheath by CLUSTER: Possible signatures of transient acceleration processes at shock. Annales Geophysicae, 21, 1483-1495. https://doi.org/10.5194/angeo-21-1483-2003

Lucek, E. A., Constantinescu, D., Goldstein, M. L., Pickett, J., Pinçon, J. L., Sahraoui, F., ... Walker, S. N. (2005). The magnetosheath. Space Science Reviews, 118, 95-152. https://doi.org/10.1007/s11214-005-3825-2

Marcucci, M. F., Bavassano Cattaneo, M. B., Pallocchia, G., Amata, E., Bruno, R., di Lellis, A. M., ... Balogh, A. (2004). Energetic magnetospheric oxygen in the magnetosheath and its response to IMF orientation: Cluster observations. Journal of Geophysical Research, 109, A07203. https://doi.org/10.1029/2003JA010312

Omidi, N., \& Sibeck, D. G. (2007). Flux transfer events in the cusp. Geophysical Research Letters, 34, L04106. https://doi.org/10.1029/2006GL028698

Palmroth, M., Hoilijoki, S., Juusola, L., Pulkkinen, T. I., Hietala, H., Pfau-Kempf, Y., ... Hesse, M. (2017). Tail reconnection in the global magnetospheric context: Vlasiator first results. Annales Geophysicae, 35, 1269-1274. https://doi.org/10.5194/angeo-35-1269-2017

Paschmann, G., Papamastorakis, I., Sckopke, N., Haerendel, G., Sonnerup, B. U. O., Bame, S. J., ... Elphic, R. C. (1979). Plasma acceleration at the Earth's magnetopause-Evidence for reconnection. Nature, 282, 243-246. https://doi.org/10.1038/282243a0

Pfau-Kempf, Y., Hietala, H., Milan, S. E., Juusola, L., Hoilijoki, S., Ganse, U., ... Palmroth, M. (2016). Evidence for transient, local ion foreshocks caused by dayside magnetopause reconnection. Annales Geophysicae, 34, 943-959. https://doi.org/10.5194/angeo-34-943-2016

Raeder, J. (2006). Flux transfer events: 1. Generation mechanism for strong southward IMF. Annales Geophysicae, 24, $381-392$. https://doi.org/10.5194/angeo-24-381-2006

Russell, C. T., \& Elphic, R. C. (1979a). ISEE observations of flux transfer events at the dayside magnetopause. Geophysical Research Letters, 6 , 33-36. https://doi.org/10.1029/GL006i001p00033

Russell, C. T., \& Elphic, R. C. (1979b). Observation of magnetic flux ropes in the Venus ionosphere. Nature, 279, 616-618. https://doi.org/10.1038/279616a0

Sibeck, D. G., \& Omidi, N. (2012). Flux transfer events: Motion and signatures. Journal of Atmospheric and Solar-Terrestrial Physics, 87, $20-24$. https://doi.org/10.1016/j.jastp.2011.07.010

Sibeck, D. G., McEntire, R. W., Lui, A. T. Y., Krimigis, S. M., \& Zanetti, L. J. (1987). The magnetosphere as a source of energetic magnetosheath ions. Geophysical Research Letters, 14, 1011-1014. https://doi.org/10.1029/GL014i010p01011

Slavin, J. A., Imber, S. M., Boardsen, S. A., DiBraccio, G. A., Sundberg, T., Sarantos, M., ... Solomon, S. C. (2012). MESSENGER observations of a flux-transfer-event shower at Mercury. Journal of Geophysical Research, 117, A00M06. https://doi.org/10.1029/2012JA017926

Southwood, D. J., Farrugia, C. J., \& Saunders, M. A. (1988). What are flux transfer events? Planetary and Space Science, 36, $503-508$. https://doi.org/10.1016/0032-0633(88)90109-2

von Alfthan, S., Pokhotelov, D., Kempf, Y., Hoilijoki, S., Honkonen, I., Sandroos, A., \& Palmroth, M. (2014). Vlasiator: First global hybrid-Vlasov simulations of Earth's foreshock and magnetosheath. Journal of Atmospheric and Solar-Terrestrial Physics, 120, 24-35. https://doi.org/10.1016/j.jastp.2014.08.012

Wei, H. Y., Russell, C. T., Zhang, T. L., \& Dougherty, M. K. (2010). Comparison study of magnetic flux ropes in the ionospheres of Venus, Mars and Titan. Icarus, 206, 174-181. https://doi.org/10.1016/j.icarus.2009.03.014 